\title{
Fatigue Limit Evaluation of Composite Materials by Means of TSA
}

\author{
by P.G. Demelio, R. De Finis, U. Galietti, D. Palumbo \\ * Politecnico di Bari, Department of Mechanics, Mathematics and Management (DMMM), Viale Japigia 182, \\ 70126, Bari
}

\begin{abstract}
Thermal methods represent a valid support to study in detail fatigue behavior of materials. Both the reduction of time and costs of the experimental campaign and the possibility to assess damage phenomena correlated to dissipative heat sources developed during fatigue.

The work aims to perform the fatigue characterization of GFRP composite materials by adopting a novel and rapid procedure for assessing endurance limit. The proposed method involves a multi-parameter analysis to attain several index related to the damage phenomena which fit well with those provided by the conventional procedures.
\end{abstract}

\section{Introduction}

Composites materials show high performance requirement in different industrial fields such as boatingyachting, aeronautical or aerospace industry [1]. In particular, a technical application of composites involves in wind turbine blades since it is required high specific stiffness, strength, and good mechanical behavior [2]. Nevertheless the strong points of using composite materials, some concerns are related to the mechanical characterization by means of fatigue tests.

In effect, to perform the fatigue tests according to the Standards test methods, requires experimental campaigns in laboratory on sample specimens or directly on large components. The standardized procedures for evaluating fatigue limit of materials result in expensive and time consuming tests because of the high number of specimens being tested $[3]$.

To meet the objective to reduce test time and costs of fatigue tests, several methods have been proposed to study rapidly and consistently the various damage phenomena [4-7]. Infrared Thermography Technique (IRT) has been exploited to better understand fatigue damage on different type of materials: such as metals and composites [8-10]. Moreover several methods and data analysis have been developed to obtain the information about the fatigue behaviour by studying heat sources generated during tests [11-15]. Graphical method to assess fatigue limit in metals has been proposed by Luong [16] and Risitano [17], by adopting the surface temperature detection in samples during a stepwise loading procedure.

In Montesano work [11] the same approach was employed for determining the fatigue limit of polymer matrix composite (PMC). In this case, the entire stress-life curve has been determined by means IRT and the results fit well with the results obtained by evaluating the conventional lifespan curve. Steinberger et al [12], adopts a lock-in thermography to perform a quantitative characterization of damage by calculation of the loss factor via the hysteretic heating.

In this way, by using IRT, other authors [18], [19] evaluate with a specific data processing of recorded infrared sequences, the damage phenomena in the material. This approach leads to the temperature signal analysis in time domain for assessing the first and the second order harmonics of the thermographic signal. It can be used to describe the nonlinear signal contents in the temperature evolution, due to the thermomechanical coupling and dissipation phenomena. Colombo et al. [20] to obtain the fatigue curve of GFRP specimens made of E-glass/epoxy with a 50\% volume fiber content, exploit this approach. Also in this case, IRT provides a good estimation of the material life in the finite life region.

In other works [21-24] the potential to identify small damage by means of Thermoelastic stress analysis (TSA) was discussed.

The well-known technique Thermoelastic Stress Analysis (TSA) is a non-contact, full field technique that provides stress maps of a component subjected to dynamic loading [21-24]. The theoretical contest concerns the thermoelastic effect that arise from cyclic loading applied to a component.

When sample undergoes to dynamic loading, small and reversible temperature changes on surface are developed and temperature changes are proportional to the first stress invariant even when the adiabatic and linear elastic conditions are achieved.

Several methods based on TSA procedures have been developed in the last years for the damage monitoring of Standard specimens and complex shaped specimens such as welded joints made of metallic materials (steel, titanium an aluminium) [25], [26]. 


\subsection{1/qirt.2016.099}

The potential of the TSA on composites has been widely discussed by Emery et al. [13] by investigating different polymer-matrix-composites with different laminate type. Fruehmann et al. [14] discuss the use of the phase signal for detecting fatigue damage at low stress amplitude, as well, is highlighted.

In this work, the fatigue behavior of GFRP is studied and the onset of damage is discussed. To do this, a novel procedure is shown for processing thermographic data capable of describing the fatigue behavior of GFRP composites and to rapidly evaluate the fatigue limit of material. The main strong point of the proposed method is related to the possibility of assessing by a single thermographic data analysis, information about the dissipative heat sources and the thermoelastic heat source.

The fatigue tests were carried out on five standard specimens made of GFRP composites material and each test has been monitored at regular interval with a cooled infrared camera. Finally, conventional fatigue tests were also carried out in order to obtain a comparison with the proposed procedure.

\section{Theoretical field}

During dynamic loading two thermal effects are generated: thermoelastic heat sources and intrinsic dissipations. The first represents the well-known thermoelastic coupling term related to dynamic loading while, intrinsic dissipation is thermodynamically irreversible. Dissipative phenomena arise due to the viscoelasto-plastic nature of the matrix material, matrix cracking, fibre fracture, and interface cracking /friction among others [11].

Whether the adiabatic conditions are achieved, the temperature changes $\Delta T_{e l}$ for orthotropic materials are related to the changes in the stresses in the material principal directions by the following expression:

$$
\Delta T_{e l}=-\frac{T_{0}}{\rho C_{p}}\left(\alpha_{1} \Delta \sigma_{1}+\alpha_{2} \Delta \sigma_{2}\right)
$$

where $\alpha_{1}$ and $\alpha_{2}$ are, respectively the coefficients of linear thermal expansion relative to the principal axes, $C_{p}$ is the specific heat at constant pressure, $\rho$ is the density, $T_{0}$ is the absolute temperature and $\Delta \sigma_{1}$ and $\Delta \sigma_{2}$ are the principal stresses.

Typical TSA acquisition systems provide usually a not radiometrically calibrated $S$ signal proportional to the peak-to-peak variation in temperature during the cyclical variation of the sum of principal stress. Usually the signal $S$ is presented as a vector, where modulus is proportional to the change in temperature due to the thermoelastic effect and the phase $\varphi$ means the angular shift between the thermoelastic and the reference signal provided by loading machine [13]. In this case, the following equation can be used:

$$
A^{*} S=\left(\alpha_{1} \Delta \sigma_{1}+\alpha_{2} \Delta \sigma_{2}\right)
$$

where $A$ is a calibration constant. In time domain the equation (2) can be expressed as follows:

$$
s=\frac{S}{2} \sin (\omega t+\pi+\varphi)
$$

where $s$ is the uncalibrated thermographic signal, $\omega$ is the angular velocity and $\varphi$ is the phase angle between temperature and loading signal. The phase angle depends on a number of parameters such as, for example, thickness of the painting or the grips of the loading machine. Whilst phase can slightly change through analysed area due to nonperfect homogeneity of the surface conditions, it remains locally constant in presence of linear elastic behaviour of material and thus, if adiabatic conditions are achieved. If damage occurs, non-linearity of thermoelastic signal and phase variations can be observed [14].

As shown in equation 3, the thermoelastic signal varies at the same frequency of the loading during the test while several authors have demonstrated that the intrinsic dissipations occur at twice the frequency of mechanical loading. Moreover the signal related to dissipations in the material are two order lower than thermoelastic ones [18].

Intrinsic dissipations are irreversible sources opposed to thermoelastic ones. The presence of irreversible phenomena affects temperature by determining an increase of mean temperature of the specimen. In particular, in presence of damage, the superficial temperature of the specimen increases, then it will tend to stabilize on a plateau value and, in the eventuality of failure occurring at a certain loading step, temperature will abruptly increase, as confirmed by several authors [15]. 


\section{Experimental Campaign}

The specimens (twelve in number) were obtained from a laminate panel made of an epoxy-type resin reinforced with the following layup $+45^{\circ} / 0^{\circ} / 45^{\circ} / 90^{\circ}$. Geometry and dimensions of specimens, obtained according to standard ASTM D 3039, were: $25 \mathrm{~mm}$ width, $250 \mathrm{~mm}$ length and thickness of $2.5 \mathrm{~mm}$. All the specimens were tested on a MTS (model 370, $100 \mathrm{kN}$ capacity) servo-hydraulic machine.

The test methods were of two types: seven samples were tested with conventional procedure in order to obtain the S-N curve ( In table 1 are shown the maximum stress applied adopting a stress ratio of 0.1 and a loading frequency of $7 \mathrm{~Hz}$ ), the second procedure was a stepwise test with the same fatigue test parameter. As shown in table 2, the loading procedure was carried out starting by a nominal stress amplitude $(\Delta \sigma / 2)$ of $30 \mathrm{MPa}$. At the end of each step (about 10.000 cycles) of loading machine the applied load was increased according to values shown in table 2. In Figure 1 represents the experimental set-up (a) and an exhausted specimen (b).

The IR camera provided a cooled In-Sb detector FLIR X6540 SC (640X512 pixel matrix array, with thermal sensitivity NETD $<30 \mathrm{mK}$ ). FLIR X6540 SC has been used either to collect the thermal data and for monitoring superficial temperature of specimens. Each loading step lasts about 20000 cycles of loading machine. During the stepwise procedure, thermal sequences were acquired during each loading step at 8000 cycles in correspondence of steady-state temperature conditions. The adopted frame rate was $100 \mathrm{~Hz}$. Each acquisition lasts $10 \mathrm{~s}$, therefore 1000 frames were recorded. Thermal sequences were analyzed by software IRTA ${ }$ and Matlab ${ }^{\circledR}$.
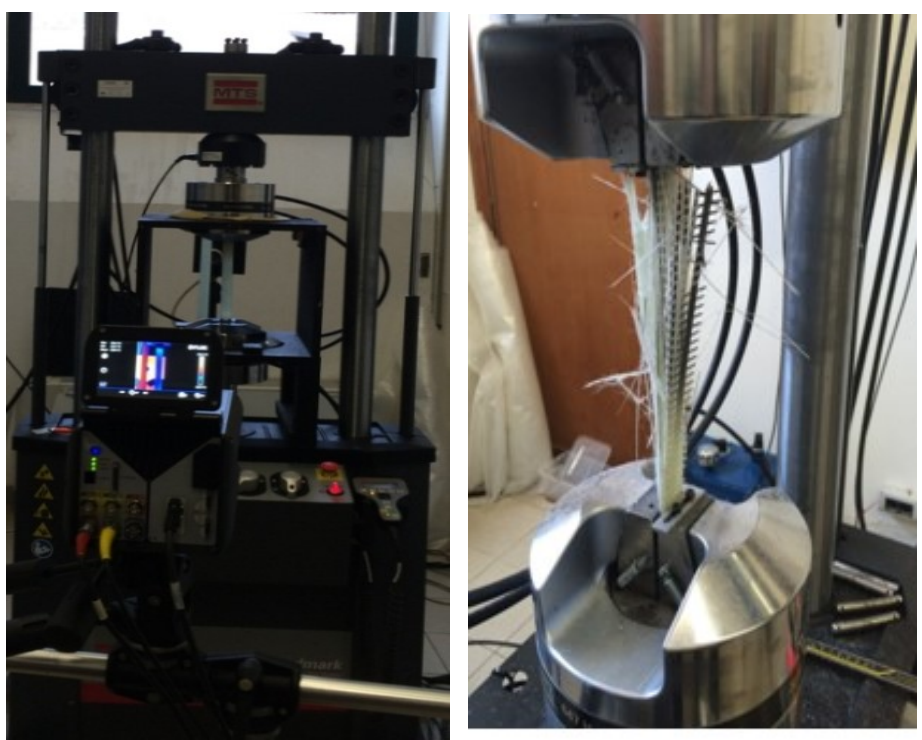

Fig. 1. Experimental set-up for thermographic tests

\section{Data Processing}

A mathematical algorithm was adopted to extract pixel by pixel information about the superficial temperature of the specimen at steady state conditions, the signal amplitude and the phase of the thermoelastic signal the amplitude of the second Fourier harmonic component related to the intrinsic dissipation. In particular, a suited thermographic signal model has been used to study the thermal signal $S_{m}$ evolution in the time domain, as indicate in equation (4):

$$
S_{m}(t)=S_{0}+a t+S 1 \sin (\omega t+\varphi)+S 2 \sin (2 \omega t)
$$

Table 1. Stresses and number of cycles to failure obtained on 7 specimens

\begin{tabular}{|c|c|c|}
\hline Specimens & 6 max [MPa] & Number of cycles \\
\hline 1 & 380 & 400 \\
\hline 2 & 270 & 2630 \\
\hline 3 & 240 & 13521 \\
\hline 4 & 200 & 52434 \\
\hline
\end{tabular}




\begin{tabular}{|c|c|c|}
\hline 5 & 175 & 120540 \\
\hline 6 & 150 & 351588 \\
\hline 7 & 138.5 & 1189803 \\
\hline
\end{tabular}

Table 2. Number of loading steps and correspondents applied stresses

\begin{tabular}{|c|c|c|c|c|}
\hline Step & $\Delta \sigma / 2[\mathrm{MPa}]$ & $6 \min [\mathrm{MPa}]$ & $6 \max [\mathrm{MPa}]$ & 6 mean $[\mathrm{MPa}]$ \\
\hline 1 & 30 & 4 & 44 & 24 \\
\hline 2 & 35 & 7 & 67 & 37 \\
\hline 3 & 40 & 8 & 78 & 43 \\
\hline 4 & 45 & 9 & 89 & 49 \\
\hline 5 & 50 & 10 & 100 & 55 \\
\hline 6 & 55 & 11 & 111 & 61 \\
\hline 7 & 60 & 12 & 122 & 67 \\
\hline 8 & 65 & 13 & 133 & 73 \\
\hline 9 & 70 & 14 & 144 & 79 \\
\hline 10 & 75 & 16 & 156 & 86 \\
\hline 11 & 80 & 17 & 167 & 92 \\
\hline 12 & 85 & 18 & 178 & 98 \\
\hline 13 & 90 & 20 & 200 & 110 \\
\hline 14 & 100 & 22 & 222 & 122 \\
\hline
\end{tabular}

where $S_{0}+$ at is the contribution at the mean temperature increase during the cyclic mechanical loading, $\omega$ is angular frequency of the mechanical imposed load, $S 1$ and $\varphi$ are respectively amplitude and phase of first harmonic component of Fourier series while, S2 represents the amplitude of the second Fourier harmonic component. In particular, the harmonic related term $S 1$ corresponds to the signal variation related to thermoelastic effect, while $S 2$ term is proportional to the amplitude of intrinsic dissipation.

IRTA® software integrates Equation (4) in the algorithm. The output of processing is an image in form of data matrix for each constant parameter. In this work a multi-parameter approach will be discussed by analysing the presented parameters capable to study damage and fatigue in composites: the index are $S_{0}, S_{1}, S_{2}, \varphi$. The processing procedure for parameters is identical. The sequence of commands was applied for each loading step and provides:

- the acquisition of the thermographic sequence. About 1000 frames were acquired for each sequence.

- $\quad$ Assessing of $S_{0}, S_{1}, S_{2}, \varphi$ pixel by pixel (IRTA® software),

- $\quad$ applying a Gaussian 2D-smoothing on data matrix obtained by Matlab® software,

- matrix windowing to refer the analysis to only gauge length area. The same data matrix for all the parameters has been considered. (A1 area, figure 2).

- For $S_{0}$, by subtracting the environmental temperature signal to the steady state temperature signal $S_{0}$ achieved during each step $\left(\Delta S_{0}\right)$, is suitable to avoid room temperature influence. Environmental temperature signal has been measured by using a dummy specimen ( $\mathrm{2} 2$ area in figure 2). Evaluating $\Delta S_{0 m a x}$ in the considered data matrix (A1 area).

- Subtracting a thermoelastic amplitude reference data matrix of the first loading step when there was no damage.

In this way, the thermoelastic variations are compared to an undamaged reference condition $\left(\Delta S_{1}\right)$.

Normalizing the thermoelastic data matrix with respect to the stress amplitude $\Delta \sigma / 2$ in order to detect the thermoelastic signal variation only due to the damage $\left(S 1_{\text {norm }}=\Delta S_{1} / \Delta \sigma / 2\right)$. 
- Evaluation of the maximum and the minimum value of the thermoelastic signal $\left(\Delta S_{1} / \Delta \sigma / 2\right)$ in order to assess the $\Delta S 1_{\text {norm }}=S 1_{\text {norm_max }} S 1_{\text {norm_min }}$ from the phase data matrix. In order to avoid isolated bad pixels due for example, to "dead pixels", values of 98 th and 2th percentile were used in this paper in place of $S 1_{\text {norm_max }}$ and $S 1_{\text {norm_min }}$ $(\Delta S 1$ norm_98-2perc).

- $\quad$ Evaluation of the maximum signal of the $S 2$ signal $(S 2 \max )$.

- Evaluation of the 98th and 2th percentile value of the thermoelastic phase signal $(\varphi)$ in order to assess the $\Delta \varphi=\varphi \_98-\varphi \_2$ from the phase data matrix, in order to avoid isolated bad pixels due for example, to "dead pixels".

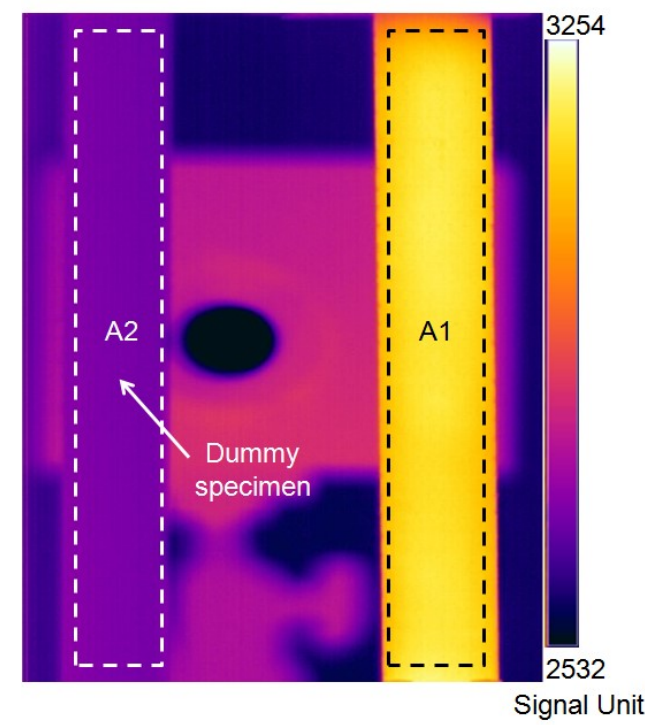

Fig. 2. Areas considered for the analysis (A1) and for evaluating of the environmental temperature signal (A2, dummy specimen)

\section{Results: Fatigue Limit Evaluation}

To assess the fatigue behaviour of the material two procedure were adopted respectively for amplitude (S0, S1, S2,) and phase $(\varphi)$ parameters. All the results have been compared with conventional S-N graph (figure 3) obtained by using the data of table 1.The S-N diagram allows for estimation of the fatigue limit of material for a conventional and fixed number of cycles of $2 * 10^{6}$ cycles. Referring to this threshold, a value of $127.4 \mathrm{MPa}$ is obtained for the fatigue limit in term of $\sigma_{\max }$ and of $56.12 \mathrm{MPa}$ in term of stress amplitude $(\Delta \sigma / 2)$.

As just said, different methods have been applied to evaluate fatigue limit due to different nature of parameters: phase shift represents the delay between load conditions and strain due to viscous behaviour in elastic and plastic stress range and damage, while amplitudes are related to absolute signal values e.g. mean temperature increase S0, stress amplitude S1, and dissipative heat sources S2. For amplitude parameters the procedure used for evaluating the fatigue limit for each measured signal ( $\Delta \mathrm{S} 0 \mathrm{max}, \Delta \mathrm{S} 1$ norm_98-2perc and $\mathrm{S} 2 \mathrm{max}$ ) is similar to the one described in the work of De Finis et al. [15]. For each specimen the adopted procedure consists in:

1. Linear regression analysis of the first 4 data couples $(P ; \Delta \sigma / 2)$ and evaluation of the best fit line $(y=m x+q)$. $P$ represent the generic thermographic signal.

2. Evaluation of residuals of $P\left(P_{r}\right)$ for each loading step.

3. Evaluation of standard deviation $\left(\sigma_{P_{-} r}\right)$ and mean $(\mu)$ of residuals $\left(P_{-} r\right)$ of the first 4 data of each tests.

4. Evaluation of the threshold value $P_{t h}=\mu+6^{*} \sigma_{P_{-} r}$

5. Evaluation of the first loading step (of $P_{-} r$ data) for which the condition: $\left(P_{-} r\right)_{N}>P_{t h}$ is verified (where $N$ is the number of the step). The first loading step exceeding the condition, is considered the estimation of fatigue limit.

Figures 4, 5 and 6 show in graphic way the procedure above exposed, on specimen 1: (a) raw data of parameters and (b) applied residual analysis to assess threshold fatigue value. In particular, the residual are plotted versus the stress amplitude and the dotted line represents the threshold value used for the estimation of the fatigue limit.

For phase data the obtained curves from processing lead to consider as fatigue limit the first point after the minimum of the curve $\Delta \varphi=\varphi_{-} 98-\varphi_{\_} 2$, figure 7 . It represents the onset of damage in the material. The typical trend of the parameter is just in debating phase, and it will be object of analysis in further work.

The results are resumed in table 3: fatigue limit values in terms of stress amplitude for each specimen and for

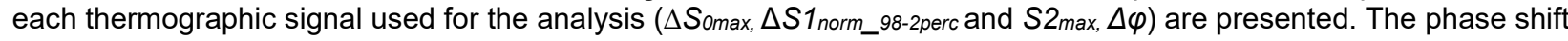
and dissipative term $S 2 \max$ seem to fit well with the S-N curve result: the values are $54.0 \mathrm{MPa}$ and 56.0 MPa respectively, comparable with 56.16 MPa the fatigue limit extrapolated out from the standard method. 


\subsection{1/qirt.2016.099}

Therefore, thermography technique allows for assessing fatigue limit values in good agreement with those obtained by adopting conventional way with a run-out limit of $2 * 10^{6}$ cycles.

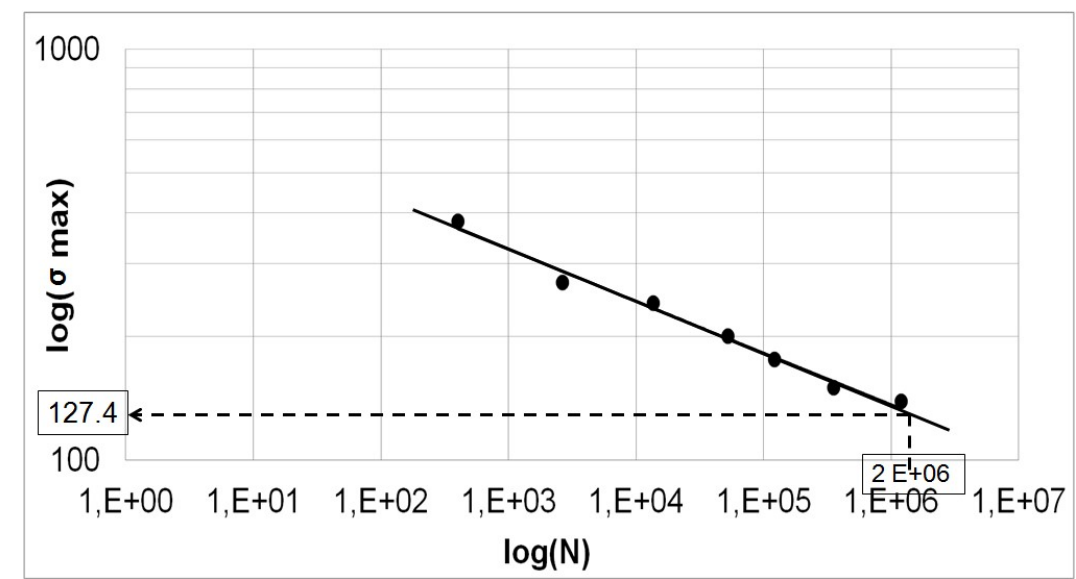

Fig. 3. Conventional S-N curve and estimation of the fatigue limit at run-out limit of $2 * 10^{6}$ cycles.

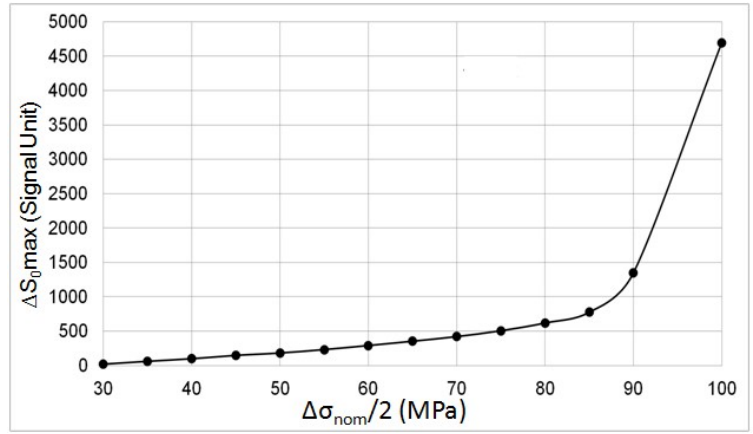

a

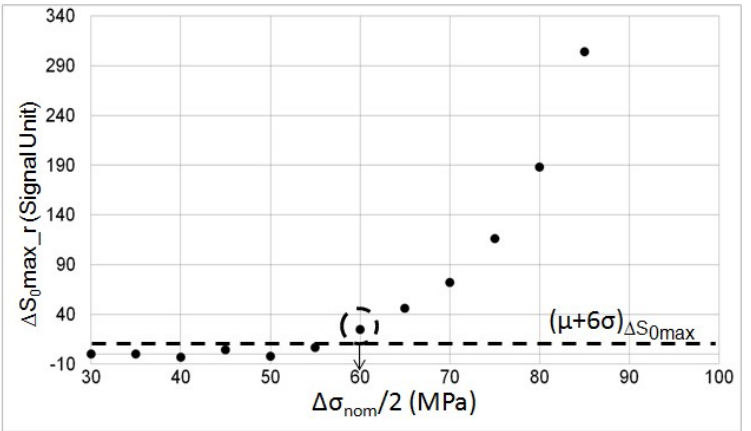

$\mathrm{b}$

Fig. 4. Specimen 1: a) Maximum of thermographic signal vs. amplitude stress and b) estimation of the fatigue limit with the method [15]

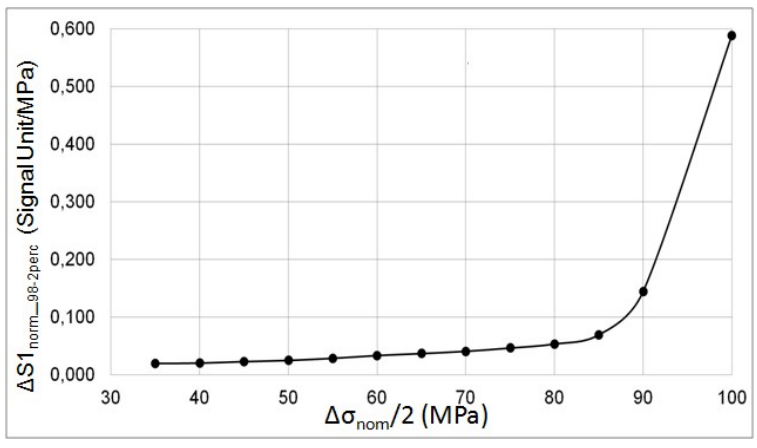

a

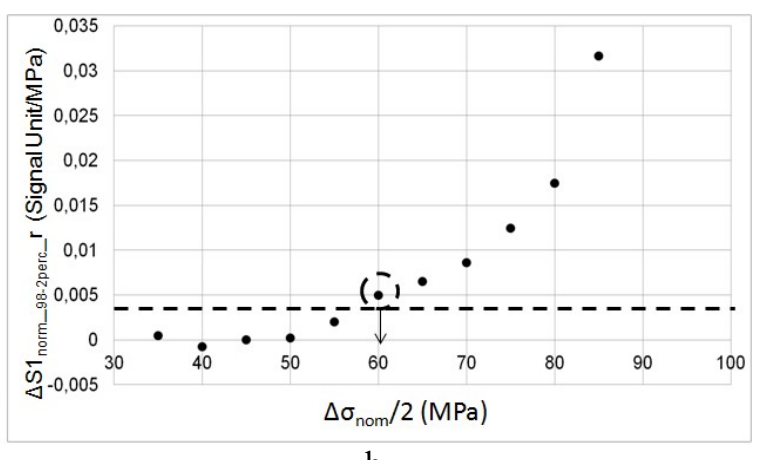

$\mathrm{b}$

Fig. 5. Specimen 1: a) Thermoelastic signal vs. amplitude stress and b) estimation of the fatigue limit with the method [15] 


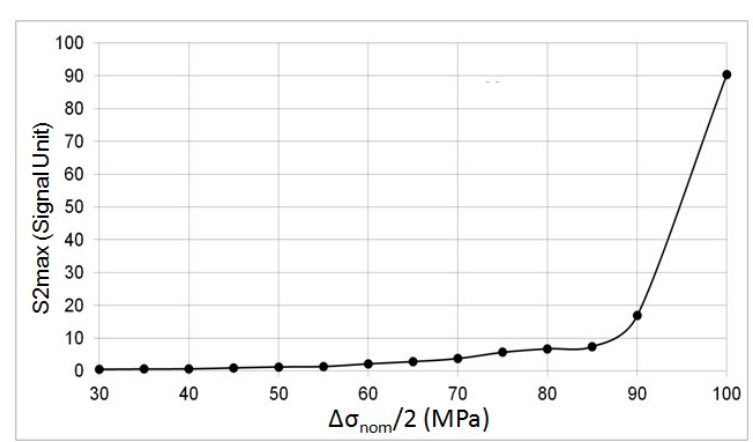

a

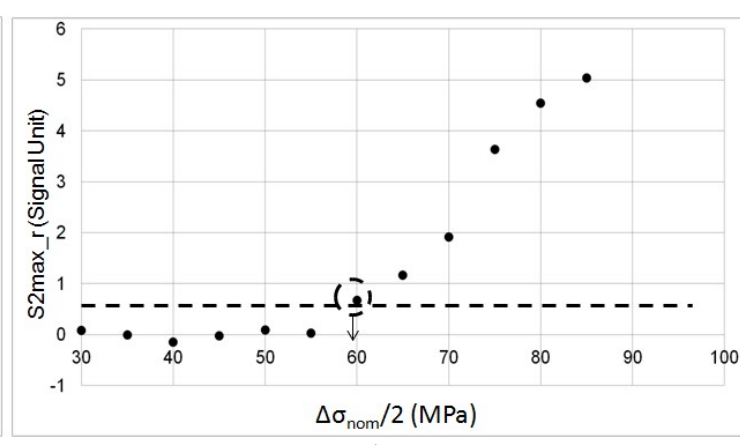

$\mathrm{b}$

Fig. 6. Specimen 1: a) Maximum thermographic signal at twice of loading frequency vs. amplitude stress and b) estimation of the fatigue limit with the method [15]



Fig. 7. Specimen 1: assessing of fatigue limit from phase shift data.

Table 3. Summary of the results obtained with the thermographic technique and comparison with the conventional fatigue tests

\begin{tabular}{|c|c|c|c|c|c|}
\hline \multicolumn{6}{|c|}{ Fatigue limit (MPa) } \\
\hline Specimens & $\Delta \boldsymbol{S}_{0 \max }$ & $\Delta S 1_{\text {norm_98-2perc }}$ & $S 2_{\max }$ & $\Delta \varphi$ & S-N curve $\left(2 * 10^{6}\right.$ cycles $)$ \\
\hline 1 & 60.0 & 60.0 & 60.0 & 60.0 & \multirow{7}{*}{$\Delta \sigma / 2=56.12$} \\
\hline 2 & 60.0 & 65.0 & 60.0 & 55.0 & \\
\hline 3 & 60.0 & 65.0 & 50.0 & 50.0 & \\
\hline 4 & 65.0 & 70.0 & 55.0 & 55.0 & \\
\hline 5 & 55.0 & 65.0 & 55.0 & 50.0 & \\
\hline Average & 60.0 & 65.0 & 56.0 & 54.0 & \\
\hline $\begin{array}{l}\text { Standard } \\
\text { deviation }\end{array}$ & 3.5 & 3.5 & 4.2 & 4.2 & \\
\hline
\end{tabular}

\section{Potential of lock-in Thermography: Damage Assessment}

The strong point of the adopted technique is represented by the possibility to assess more information related to the damage from a single thermal signal sequence.

In figure 8 the maps of thermoelastic signal are shown as function of the applied stress amplitude for the specimen 1. It has to be underlined a signal variation from positive to negative values with respect to the reference stress condition of $30 \mathrm{MPa}$. As already demonstrated in other works [13], [14], TSA leads to localize the damaged areas of material. In particular, the thermoelastic signal variations are related to the redistribution of the stresses caused by the stiffness degradation due to the damage.

In figure 9 are shown the maps of the thermal signal at twice of the loading frequency (specimen 1). Even in this case, a significant increasing of the signal is obtained as the applied stress increases.

In figure 10 the phase maps are depicted at different loading levels under/above the fatigue limit. 


\subsection{1/qirt.2016.099}

These maps seem provide different and complementary information about the damage of material if compared with the thermoelastic maps. In this regard, further works are necessary to relate the different damage mechanisms to the thermographic analysis. Starting from $75 \mathrm{MPa}$ in stress map (figure 8) and thermoelastic signal at twice the mechanical frequency (figure 9) the damage seems occur, in fact it could affect the fiber in the right side of the gage length.

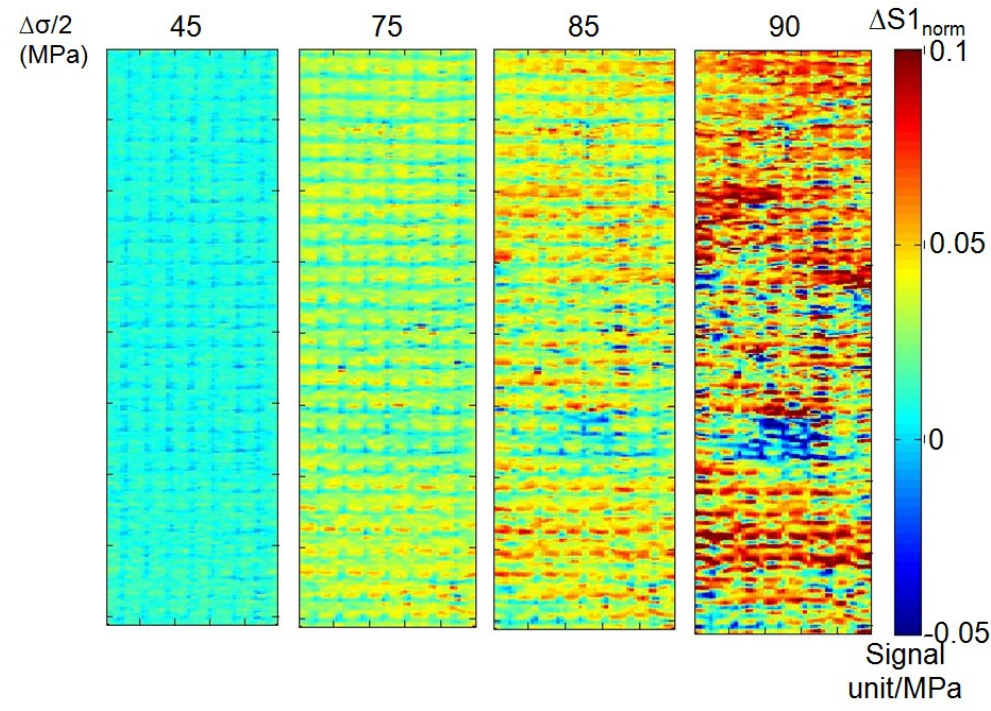

Fig. 8. Maps of the thermoelastic signal obtained at different load levels.

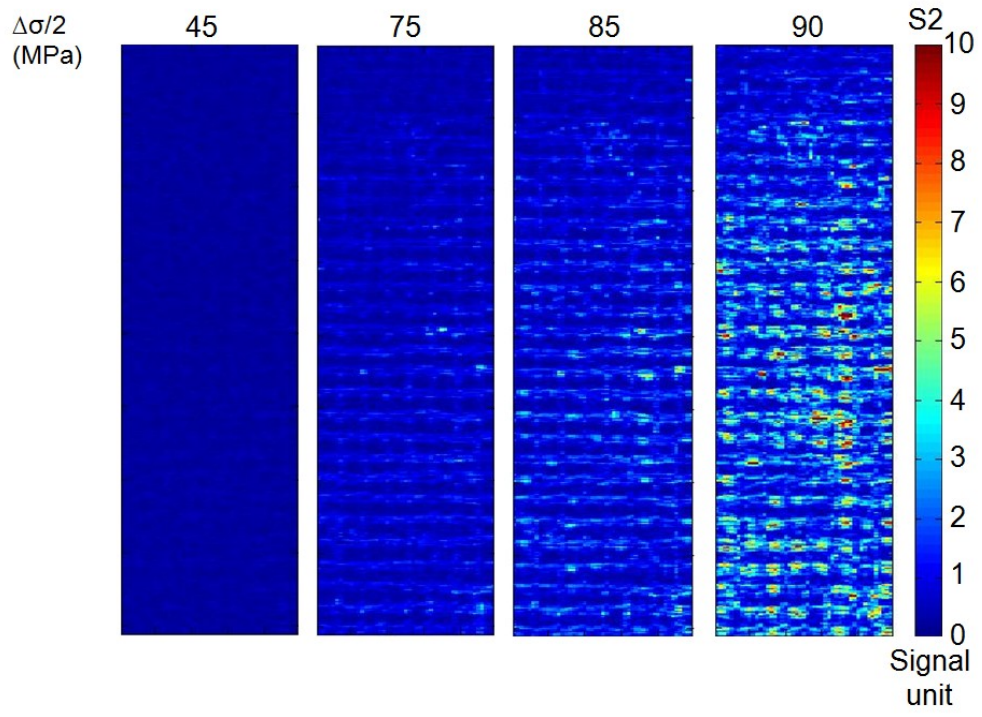

Fig. 9. Maps of the thermographic signal at the twice of the loading frequency obtained at different load levels. 

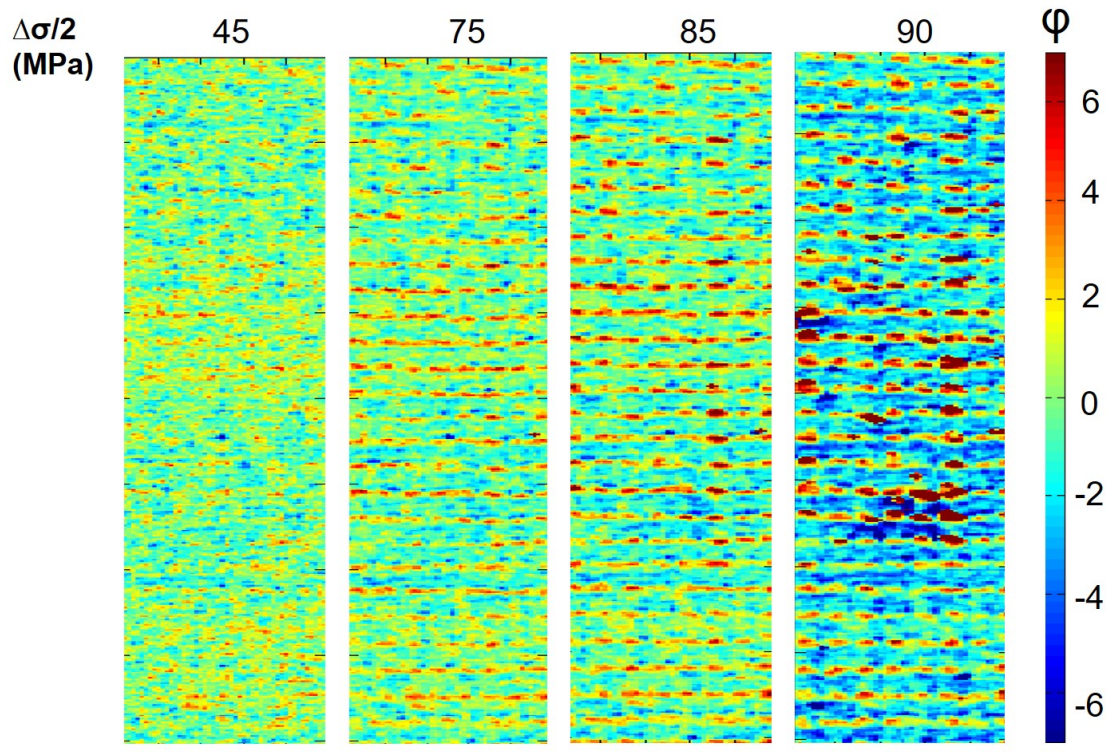

$\left[{ }^{\circ}\right]$

Fig. 10. Maps of the phase shift at different load levels.

\section{Conclusions}

A novel procedure has been proposed for evaluating the fatigue limit of GFRP composite materials with thermographic technique. In particular, the uncalibrated signal has been analyzed in the time domain for extracting information related to the temperature increase, thermoelastic, dissipative sources and phase shift between imposed stress and strain.

The potential of the technique is related to the possibility of reducing testing time: from one thermographic sequence different index to study fatigue can be assessed.

Five specimens were used for the fatigue tests and each specimen has been subjected to a loading step procedure until the failure. Two methods have been presented for determining fatigue limit, in particular one is a statistical method, validated for metallic materials. A graphical method was adopted on phase data to assess fatigue limit. Since results show a good agreement with those obtained by the conventional S-N curve, proposed procedure could represent a useful tool for the monitoring of real and more complex components subjected to actual loading conditions.

\section{REFERENCES}

[1] Bannister MK., Development and application of advanced textile composites. Proceedings of the Institution of Mechanical Engineers, Part L: Journal of Materials Design and Applications. - Vol. 218, pp. 253-260, 2004.

[2] Palumbo D., Tamborrino R., Galietti U., Aversa P., Tatì A., Luprano V.A.M., Ultrasonic analysis and lock-in thermography for debonding evaluation of composite adhesive joints. NDT \& E International. - Vol. 78, pp. 1-9, 2016.

[3] Harris B., Fatigue in composites. Cambridge: Woolhead Publishing Ltd, 2003.

[4] Munoz V., Valès B., Perrin M., Pastor M.L., Welemane H., Cantarel A., Damage detection in CFRP by coupling acoustic emission and infrared thermography. Composites: Part B. - Vol. 85, pp.68-75, 2016.

[5] Goidescu C., Welemane H., Garnier C., Fazzini M., Brault R., Péronnet E., Mistou S., Damage investigation in CFRP composites using full-field measurement technique: Combination of digital image stereo-correlation, infrared thermography and X-ray tomography. Composites: Part B. - Vol. 48, pp. 95-105, 2013.

[6] Naderi M., Kahirdeh A., Khonsari M.M., Dissipated thermal energy and damage evolution of Glass/Epoxy using infrared thermography and acoustic emission. Composites: Part B. - Vol. 43, pp.1613-1620, 2012.

[7] Kordatos E.Z., Aggelis D.G., Matikas T.E., Monitoring mechanical damage in structural materials using complimentary NDE techniques based on thermography and acoustic emission. Composites: Part B. Vol. 43, pp. 2676-2686, 2012. 


\subsection{1/qirt.2016.099}

[8] Palumbo D., Ancona D., Galietti U., Quantitative damage evaluation of composite materials with microwave thermographic technique: feasibility and new data analysis. Meccanica. - Vol. 50, pp. 443-459, 2015.

[9] Galietti U., Dimitri R., Palumbo D., Rubino P., "Thermal analysis and mechanical characterization of GFRP joints". In: 15th European Conference on Composite Materials: Composites at Venice, ECCM 2012, Venice, Italy, 24-28 June, 2012.

[10] Tamborrino R., Palumbo D., Galietti U., Aversa P., Chiozzi S., Luprano V.A.M., Assessment of the effect of defects on mechanical properties of adhesive bonded joints by using non destructive methods. Composites Part B. - Vol. 91, pp. 337-345, 2016.

[11] Montesano J., Fawaz Z., Bougherara H., Use of infrared thermography to investigate the fatigue behavior of a carbon fiber reinforced polymner composite. Composite Structures. - Vol. 97, pp. 76-83, 2013.

[12] Steinberger R., Valadas Leitão T.i., Ladstätter E., Pinter G., Billinger W., Lang R.W., Infrared thermographic techniques for non-destructive damage characterization of carbon fibre reinforced polymers during tensile fatigue testing. International Journal of Fatigue. - Vol. 28, pp. 1340-1347, 2006.

[13] Emery T.R., Dulieu-Barton J.K., Thermoelastic Stress Analysis of the damage mechanisms in composite materials. Composites: Part A. - Vol. 41, pp. 1729-1742, 2010.

[14] Fruehmann R.K., Dulieu-Barton J.M., Quinn S., Assessment of the fatigue damage evolution in woven composite materials using infra-red techniques. Composite Science and Technology. Vol. 70, pp. 937-946, 2010.

[15] De Finis R., Palumbo D., Ancona F., Galietti U., Fatigue Limit Evaluation of Various Martensitic Stainless Steels with New Robust Thermographic Data Analysis. International Journal of Fatigue. - Vol. 74, pp. 88-96, 2015.

[16] Luong M.P., "Infrared observation of thermomechanical couplings in solids". Thermosense XXIV Conference, part of SPIE's Aerosense 1-Orlando (Florida), 5 April, 2002.

[17] La Rosa G., Risitano A., Thermographic methodology for the rapid determination of the fatigue limit of materials and mechanical components, International Journal of Fatigue. - Vol. 22, pp. 65-73, 2000.

[18] Krapez JK., Pacou D., Gardette G., Lock-In Thermography and Fatigue Limit of Metals. Quantitative Infrared Thermography. - Vol. 6, pp. 277-282, 2000.

[19] Ummenhofer T., Medgenberg J., On the Use of Infrared Thermography for the Analysis of Fatigue Damage Processes in Welded Joints. International Journal of Fatigue. - Vol. 31, pp. 130-137, 2009.

[20] Colombo C., Libonati F., Pezzani F., Salerno A., Vergani L., Fatigue behaviour of a GFRP laminate by thermographic measurements. Procedia Engineering. - Vol. 10, pp.3518-3527, 2011.

[21] Harwood N., Cummings W., "Thermoelastic stress analysis". New York: National Engineering Laboratory; Adam Hilger, 1991.

[22] Pittaresi G., Patterson E.A., A review of the general theory of thermoelastic stress analysis. Journal of Strains Analysis. - Vol. 35, pp. 35-39, 1999.

[23] Wang W.J., Dulieu-Barton J.M., Li Q., Assessment of Non-Adiabatic Behaviour in Thermoelastic Stress Analysis of Small Scale Components, Experimental Mechanics. - Vol. 50, pp.449-461, 2010.

[24] Palumbo D., Galietti U., Data Correction for Thermoelastic Stress Analysis on Titanium Components, Experimental Mechanics. - Vol. 56, pp. 451-462, 2016.

[25] Palumbo D., Galietti U., Characterization of Steel Welded Joints by Infrared Thermographic Methods, Quantitative Infrared Thermography Journal. - Vol. 11, no 1, pp. 29-42, 2014.

[26] Galietti U., Palumbo D., "Application of thermal methods for characterization of steel welded joints". EPJ Web of Conferences, 6: 38012, 2010. 